\title{
HIGH STABILITY MICROWAVE FREQUENCY DISTRIBUTION WITH OPTICAL FIBER
}

\author{
Yi Dong*, Lumin Zhang, Le Chang, Zhaohui Li, Lilin Yi, Weisheng Hu \\ * State Key Laboratory of Advanced Optical Communication Systems and Networks, \\ Shanghai Jiao Tong University, Shanghai, 200240, China \\ Phone: +(021)34204390, Fax: +(021)34204390, Email: yidong@sjtu.edu.cn
}

Keywords: microwave frequency distribution, optical fiber, opto-electronic delay locked-loop

\section{Experiment}

\begin{abstract}
We improve the phase stabilization of remote RF distribution based on opto-eletronic delay locked-loop with a different wavelength backward signal. RMS jitter measured at remote end is under $730 \mathrm{fs}$ for a $10 \mathrm{GHz}, 100 \mathrm{~km}$ transmission.
\end{abstract}

\section{Introduction}

Remote transmission of phase-stabilized microwave frequency is a key technology in very long baseline interferometer and precision clock synchronization $[1,2]$. Traditional method for transfer frequency and time standards over long distances is the common-view global positioning satellite system (GPS). But GPS techniques do not provide the short-term stability necessary for synchronization applications. In recent years, with the attractive features like low loss, high bandwith and immunity to electromagnetic interference, optical fiber has been a promising alternative for remote frequency reference transmission. However, pressure and temperature variation along the fiber link results in the accumulative phase fluctuation, degrading the frequency phase stability at the remote end. Therefore, a real-time remote phase detection and drift cancellation system are required. The so-called round trip phase correction is a basic method in remote phase control systems. By detecting the phase of the round back signal at local, the remote phase variation is measured and then corrected by the servo system[3].

In our previous work [4], we proposed a remote phase drift cancellation system based on a heterodyne opto-electronic delay locked loop, and demonstrated the concept by transmitting a $10-\mathrm{GHz}$ microwave frequency over $50 \mathrm{~km}$ single mode fiber (SMF), acquiring sub pico-second jitter at the remote end. Recently, we incorporate an active optical regeneration at the remote end, slightly shifting the optical wavelength of the backward signal for approximately $3 \mathrm{~nm}$. The wavelength difference eliminates the coherent Rayleigh noise induced by the interference between backward signal and the Rayleigh back scattering of the forward signal, therefore reduces the signal to noise ratio (SNR) of the round back signal. With this improvement, the transmission distance is extended to $100 \mathrm{~km}$ with remote jitter measured under $730 \mathrm{fs}$.

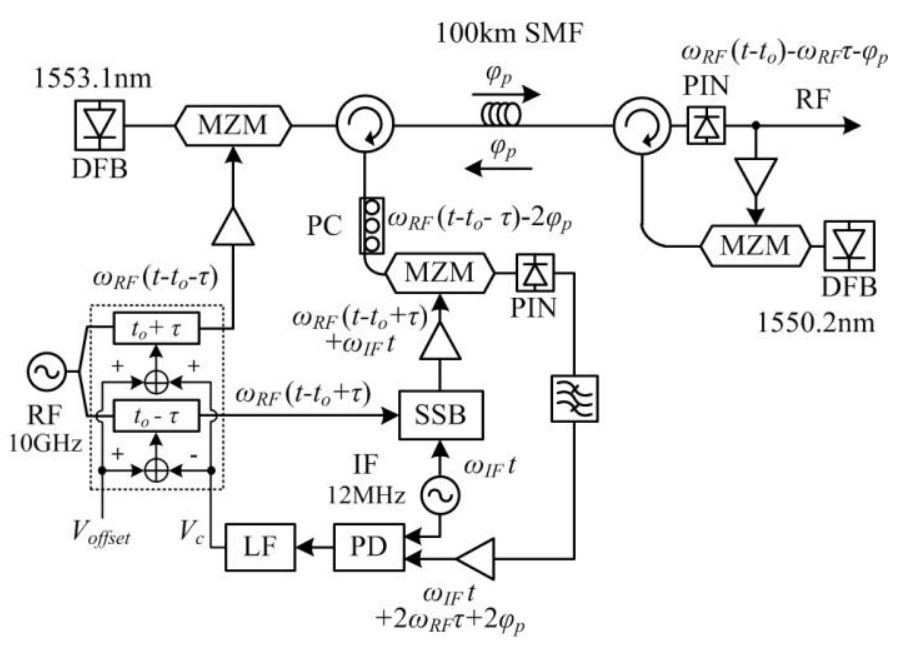

Fig. 1. Experiment setup for the $100 \mathrm{~km}$ phase drift cancellation system.

The experimental setup is outlined in fig. 1, with the local control system similar to the corresponding part in our previous work. The $10 \mathrm{GHz}$ microwave local oscillator (LO) provided by a radio frequency signal generator is divided to two branches, being delayed separately in the microwave delay module. Delay periods are controlled by voltage which equal to the sum and difference between a fixed offset voltage and the loop filter (LF) output respectively. First branch of LO is amplified to $V_{\pi}$ of a Mach-Zender modulator (MZM) to amplitude modulate a narrow linewidth $(3 \mathrm{kHz}) \mathrm{CW}$ distributed feedback (DFB) laser running at $1553 \mathrm{~nm}$. The modulated carrier is sent to remote receiver via a coiled $100 \mathrm{~km}$ G.652 single mode fiber in laboratory. At the remote end, the optical signal is received by a photodiode, amplified and then re-drives the MZM in remote end to modulate the backward optical carrier. Wavelength of the carrier is set to $1550 \mathrm{~nm}$ to avoid the coherent Rayleigh noise.

Backward signal routed by the circulator is modulated through the second local MZM. The polarization status of the round back signal is adjusted by a polarization controller (PC). Second branch of microwave LO is frequency shifted for an intermediated frequency (IF) through a single sideband modulator (SSB). The IF signal is generated by a $12-\mathrm{MHz}$ crystal and divided to two branches, one to feed the SSB 
modulator and the other to provide a local IF reference. The frequency shifted LO is amplified to $2 V_{\pi}$ to drive the second local MZM with a bias at the transmission null point, which maximizes the modulation nonlinearity of MZM. This results in the frequency mixing between the round back signal and the frequency shifted LO. Therefore, phase information of the round back signal is identically down-converted to IF. This IF phase signal is received by a low speed photodetector, amplified and filtered by the electronic circuits. The phase detector (PD) discriminates the phase difference between the received IF signal and the local reference source. Output of the phase detector is integrated in the loop filter (LF) and sent back to control the microwave delay module.

Phase relationship in the control loop is illustrated in Fig.1. Given that the angular frequency of the microwave LO is $\omega_{R F}$, after propagation, remote signal can be expressed by Eq.1, where $t_{o}$ represents the fixed delay due to offset voltage and $\tau$ is controlled by LF output. $\varphi_{P}$ is the phase shift induced by the transmission fiber. Higher order harmonics in the MZM transfer function and amplitude factors are ignored the in the equation.

$$
E_{\text {remote }}(t)=\cos \left[\omega_{R F}\left(t-t_{o}\right)-\omega_{R F} \tau-\varphi_{P}\right]
$$

Since the optical signals passing through the fiber in both directions experience the same phase perturbations, the same phase shift is included in the round back signal. When driven by a frequency shifted microwave LO, the modulation nonlinearity of MZM results in the frequency mixed operation, and generates an IF signal shown in Eq. 2.

$$
E_{I F_{-} \text {receive }}(t)=\cos \left[\omega_{I F} t+2\left(\omega_{R F} \tau+\varphi_{P}\right)\right]
$$

The phase detector produces an error signal when there is phase difference between the received IF signal and the local IF reference, which is integrated in LF to control the microwave delay module. Therefore, delay locked loop maintains the same phase for the received IF signal as the IF reference, by adjusting the delay period when the propagation phase fluctuates. Namely, the phase variation component $\left(\omega_{R F} \tau+\varphi_{P}\right)$ is kept zero at both received IF signal and remote microwave, therefore the phase drift of the remote RF signal was canceled, and the remote phase is stabilized.

We measure the root mean square (RMS) phase jitter at the local and remote receiver with a digital sampling oscillator (Tektronix model TDS8200). The jitters are measured at different periods and plotted in fig. 2 . The measured jitter of the previous $50 \mathrm{~km}$ transmission is also plotted in the same figure for comparison. RMS jitter of locally received signal is measured between $702 \mathrm{fs}$ and $720 \mathrm{fs}$, which indicates the intrinsic system jitter level of the back-to-back system. Benefit from the different wavelength backwards, the RMS jitter measured at remote end of $100 \mathrm{~km}$ transmission is under $730 \mathrm{fs}$, more than $100 \mathrm{fs}$ improvement compared with the 50$\mathrm{km}$ transmission with passive remote end.

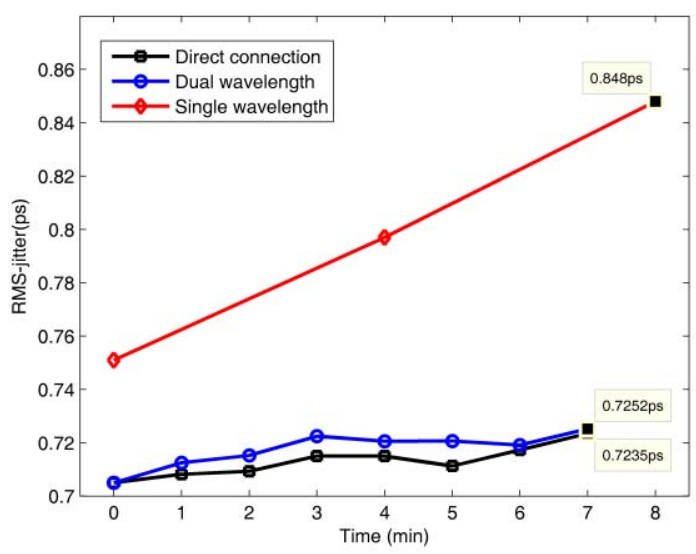

Fig. 2 Measured receiver root mean square (RMS) phase jitter at different period, in three conditions: locally, $50 \mathrm{~km}$ transmission with same wavelength backward, $100 \mathrm{~km}$ transmission with different wavelength backward.

\section{Conclusion}

In summary, we improve the jitter performance of our previously proposed remote phase stabilization method based on opto-electronic delay locked loop. With an active remote end and different wavelength sent back to the local end, the transmission distance is extended to $100 \mathrm{~km}$, and remote RMS jitter is reduced to $730 \mathrm{fs}$. Though with an increased the system complexity, the signal regeneration scheme at remote end shows better performance especially in long distance transmission.

\section{Acknowledgements}

This work was supported by the National Basic Research Program of China (973 Program) (2012CB315602) and the National Natural Science Foundation of China (NSFC) (61027007).

\section{References}

[1] K. H. Sato, T. Hara. "Application of phase-stabilized optical fiber in transmission of reference and IF signals in VLBI observation ", IEEE Trans. Instrum. Meas. , 41, pp. 385-389, (1992).

[2] Seth M. Foreman, Kevin W. Holman, Darren D. Hudson, David J. Jones, and Jun Ye "Remote transfer of ultrastable frequency references via fiber networks", REVIEW OF SCIENTIFIC INSTRUMENTS 78, 0211011-25 (2007).

[3] Narbonneau, F., Lours, M., Bize, S., et al. High resolution frequency standard dissemination via optical fiber metropolitan network [J]. Review of Scientific Instruments: 2006, 77(6): 064701.

[4] Lumin Zhang, Le Chang, Yi Dong, Weilin Xie, Hao He, and Weisheng $\mathrm{Hu}$ "Phase drift cancellation of remote radio frequency transfer using an optoelectronic delaylocked loop", Optics Letters, 36, pp. 873-875, (2011). 